\title{
The interaction of transient receptor potential melastatin 7 with macrophages promotes vascular adventitial remodeling in transverse aortic constriction rats
}

\author{
Yan $\mathrm{Li}^{1}$, Hui Jiang ${ }^{2}$, Chengchao Ruan ${ }^{1}$, Jiuchang Zhong ${ }^{1}$, Pingjin Gao ${ }^{1}$, Dingliang Zhu ${ }^{1}$, Wenquan Niu ${ }^{1}$ \\ and Shujie Guo ${ }^{1}$
}

Transient receptor potential melastatin 7 (TRPM7), a novel channel kinase, has been recently identified in the vasculature. However, its regulation and function in vascular diseases remain poorly understood. To address this lack of knowledge, we sought to examine whether TRPM7 can mediate the vascular remodeling process induced by pressure overload in the right common carotid artery proximal to the band (RCCA-B) in male Sprague-Dawley rats with transverse aortic constriction (TAC). The contribution of TRPM7 to amplified vascular remodeling after TAC was tested using morphometric and western blot analyses. Pressure overload-induced vascular wall thickening, especially in the adventitia, was readily detected in RCCA-B. The TRPM7 level was increased with a simultaneous accumulation of macrophages in the adventitia of RCCA-B, whereas the anti-inflammatory molecule annexin-1, a TRPM7 downstream target, was decreased. After the addition of the TRPM7 inhibitor 2-aminoethoxydiphenyl borate (2-APB), significant reductions in macrophage accumulation as well as the expression of monocyte chemotactic protein-1, SM-22- $\alpha$ and collagen I were observed, whereas annexin-1 was rescued. Finally, in cultured vascular adventitial fibroblasts treated with macrophage-conditioned medium, there were marked increases in the expression of TRPM7 and SM-22- $\alpha$ with a concurrent reduction in annexin-1 expression; these effects were largely prevented by treatment with 2-APB and specific anti-TRPM7 small interfering RNA. Our findings provide the first demonstration of the potential regulatory roles of TRPM7 in the vascular inflammation, pressure overload-mediated vascular adventitial collagen accumulation and cell phenotypic transformation in TAC rats. The targeting of TRPM7 has potential therapeutic importance for vascular diseases.

Hypertension Research (2014) 37, 35-42; doi:10.1038/hr.2013.110; published online 12 September 2013

Keywords: adventitia; annexin-1; macrophage; transient receptor potential melastatin 7; transverse aortic constriction

\section{INTRODUCTION}

Thickening of the arteries has been implicated in the development of hypertension. ${ }^{1,2}$ Recently, the contributory roles of adventitia in artery remodeling have garnered increasing attention. For example, it has been well documented that adventitial fibroblasts (AFs) can be transformed into myofibroblasts (MFs) and migrate into the neointima of arteries after endoluminal injury in balloon injury models. ${ }^{3-6}$ Our recent findings and those of others using a transverse aortic constriction (TAC) model collectively showed that the arteries under pressure overload experience dramatic remodeling processes including changes in morphology, vessel composition and mechanical properties. ${ }^{7-9}$ The induced pressure overload in the TAC model can mimic the status of arteries under volume-dependent hypertension in humans, although, unfortunately, an ideal animal model for adventitial remodeling is not yet available. Therefore, the elucidation of the underlying remodeling mechanisms remains a challenging task.

Transient receptor potential melastatin 7 (TRPM7), an Mg-ATPregulated divalent cation channel with a unique bifunctional structure (viz. the ion channel and carboxyl-terminal $\alpha$-kinase domain), was first identified in 2001. ${ }^{10,11}$ The role of TRPM7 in regulating $\mathrm{Mg}^{2+}$ homeostasis has been well established in hypertension. ${ }^{12,13}$ TRPM7 can mediate proinflammatory bradykinin signaling where annexin-1 has been activated. ${ }^{14,15}$ As one of the identified substrates for TRPM7, annexin-1 executes its anti-inflammatory impacts by modulating the expression of inflammatory factors and the chemotaxis of

\footnotetext{
${ }^{1}$ State Key Laboratory of Medical Genomics, Shanghai Key Laboratory of Hypertension, Ruijin Hospital, Shanghai Jiao Tong University School of Medicine, Shanghai, China and ${ }^{2}$ Laboratory of Vascular Biology, Institute of Health Sciences, Shanghai Institute for Biological Sciences, Chinese Academy of Sciences, Shanghai, China

Correspondence: Dr S Guo, or Dr W Niu, State Key Laboratory of Medical Genomics, Shanghai Key Laboratory of Hypertension, Ruijin Hospital, Shanghai Jiao Tong University School of Medicine, Shanghai, China. 
inflammatory cells. ${ }^{16-18}$ In a recent study, we detected lower expression of annexin-1 in both AFs and vascular adventitia of spontaneously hypertensive rats (SHR) as compared with Wistar-Kyoto rats. ${ }^{19} \mathrm{~A}$ growing body of evidence suggests that TRPM7 variation, along with altered annexin-1, alters the function of the vascular endothelial and smooth muscle cells. ${ }^{14,15,20-22}$ Therefore, it is of added interest to test whether TRPM7, an upstream molecule of annexin-1, is associated with the remodeling process. To address these issues, we sought to examine whether TRPM7 can mediate the vascular remodeling process induced by pressure overload in the RCCA-B in TAC rats.

\section{METHODS}

\section{Animal model}

Male Sprague-Dawley rats $(270 \pm 20 \mathrm{~g})$ were obtained from the Shanghai SLAC Laboratory Animal (Shanghai, China). TAC was induced as described previously. ${ }^{23}$ In brief, rats were anesthetized with an intraperitoneal injecting mixture of ketamine $\left(100 \mathrm{mg} \mathrm{kg}^{-1}\right)$ and xylazine $\left(5 \mathrm{mg} \mathrm{kg}^{-1}\right)$ and were connected to a volume-cycled rodent ventilator at a respiratory rate of 70 breaths per minute and a tidal volume of $2.5 \mathrm{ml}$. The thoracic cavity was opened, and the aorta was ligated between the origins of the innominate and left carotid arteries. In sham-operated rats, a suture was placed around the aorta but not tightened. To inhibit TRPM7, 2-aminoethoxydiphenyl borate (2-APB, $2.5 \mathrm{mg} \mathrm{kg}^{-1}$; Sigma-Aldrich, Shanghai, China) was administered to the rats by intraperitoneal injection once every 3 days. The first injection was administered at $1 \mathrm{~h}$ before the TAC operation. 2-APB was first dissolved in dimethyl sulfoxide to $50 \mu \mathrm{g} \mu \mathrm{l}^{-1}$ and then diluted with a saline solution to $0.5 \mu \mathrm{gl}^{-1}$ (1:100 dilution). All procedures were performed in accordance with the Care and Use Guide of Laboratory Animals of the National Institutes of Health with the approval (SYXK-2003-0026) of the Scientific Investigation Board of Shanghai Jiao Tong University School of Medicine.

\section{Histological analysis}

Rats were killed postoperatively at 14th day after perfusion with $4 \%$ paraformaldehyde under physiological pressure. The right carotid artery was carefully removed and embedded in optimum cutting temperature tissuefreezing medium (Sakura, Torrance, CA, USA) or in paraffin after formalin fixation for $24 \mathrm{~h}$. For morphometric analysis, paraffin-embedded tissue was sectioned at $5 \mu \mathrm{m}$ thickness and stained with hematoxylin and eosin followed by examination by light microscopy (Carl Zeiss, Jena, Germany). The medial and adventitial cross-section areas were measured using Axio Vision 4.8.1 software (Carl Zeiss).

Frozen arteries embedded in the optimum cutting temperature compound were sectioned at $10 \mu \mathrm{m}$ and mounted on glass slides. The slides were air-dried, acetone-fixed and blocked with $5 \%$ bovine serum albumin followed by incubation with primary antibodies to CD68 (1:200; Serotec, Oxford, UK), TRPM7 (1:100; Abcam, Cambridge, UK), annexin-1 (1:100; Santa Cruz Biotechnology, Santa Cruz, CA, USA), SM-22- $\alpha$ (1:200; Abcam), monocyte chemotactic protein-1 (MCP-1; 1:100; Santa Cruz Biotechnology) and collagen I (1:100; Santa Cruz Biotechnology), and then secondary antibodies conjugated with fluorescein isothiocyanate or tetrametrylrhodarnine isothiocyante (Invitrogen, Carlsbad, CA, USA). Nuclei were stained with 4'-6-diamidino-2-phenylindole (Sigma-Aldrich, Saint Louis, MO, USA). Sections were mounted in fluorescence-mounting medium (Dako, Copenhagen, Danmark). Laser scanning confocal microscopy was performed using a Zeiss epifluorescence microscope system.

\section{Cell culture}

The methods used for the isolation and culture of AFs from thoracic aorta of 6-8-week-old male Sprague-Dawley rats were similar to previously described techniques. ${ }^{24}$ Briefly, adventitia of thoracic aortas were separated and cut into sections, which were incubated on dishes with Dulbecco's modified Eagle medium (DMEM) containing 10\% fetal calf serum (Gibco, Grand Island, NY, USA), $100 \mathrm{U} \mathrm{ml}^{-1}$ penicillin and streptomycin. Subconfluent cells were made quiescent by incubation in DMEM supplemented with $0.1 \%$ bovine serum albumin for $24 \mathrm{~h}$ before stimulation. Cells were used from passages $4-6 .{ }^{25}$ The mouse promyelocytic leukemia cell line RAW264.7 was purchased from Cell Bank of the Chinese Academy of Sciences (Shanghai, China). RAW264.7 cells were grown in DMEM containing with $10 \% \mathrm{FBS}$ at $37^{\circ} \mathrm{C}$ in $5 \% \mathrm{CO}_{2}$. Subconfluent cells were incubated with serum-free DMEM medium, and the conditioned medium was collected after $48 \mathrm{~h}$.

AFs were treated with $100 \%$ macrophage-conditioned medium for $6,12,24$ and $48 \mathrm{~h}$ and were incubated for $24 \mathrm{~h}$ in $1-100 \%$ macrophage-conditioned medium. To inhibit TRPM7, AFs were pretreated with 100- $\mu$ M 2-APB for $1 \mathrm{~h}$ and then stimulated with 100\% RAW264.7-conditioned medium for an additional $24 \mathrm{~h}$. 2-APB was first dissolved in dimethyl sulfoxide to $0.2 \mathrm{M}$ and then diluted with DMEM- or RAW264.7-conditioned medium to $100 \mu \mathrm{M}$ (1:2000 dilution). It has been reported that a dimethyl sulfoxide level of $<0.1 \%$ does not have any effect of its own, ${ }^{26}$ and thus solvent control was not performed in this present study.

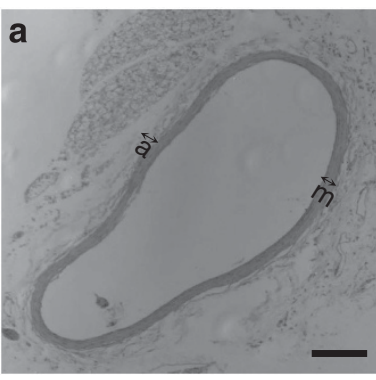

SHAM

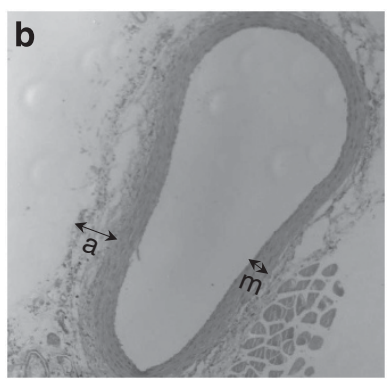

TAC
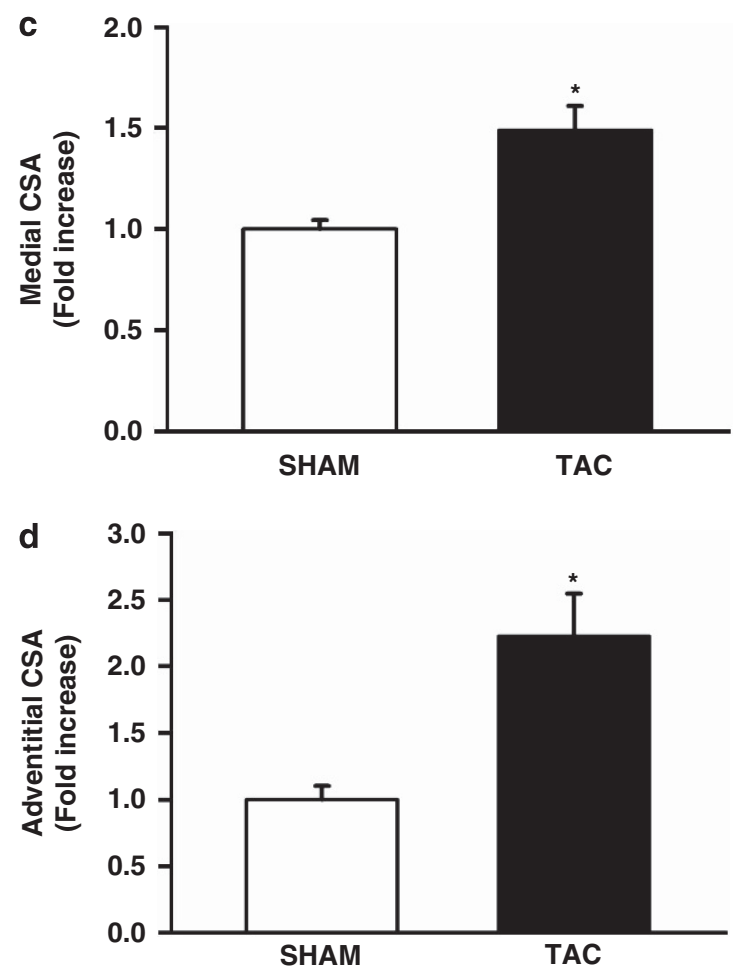

Figure 1 Histomorphometric analysis of the right common carotid artery proximal to the band (RCCA-B) in the sham-operated (SHAM) and transverse aortic constriction (TAC) groups 14 days after the procedure. (a,b) Hematoxylin and eosin staining revealed increased medial and adventitial thicknesses in RCCA-B from the TAC group. a, adventitia; $m$, media. Bar, $100 \mu \mathrm{m}$. Total magnification, $\times 100$. (c,d) Quantification data showing increased folds of the medial and adventitial cross-section areas in the TAC group. Six sections were used in the quantification. Results are expressed as the mean \pm s.d., $n=6$ for each group; ${ }^{*} P<0.05$ compared with the SHAM group. A full color version of this figure is available at Hypertension Research online. 


\section{siRNA and transfection}

The anti-TRPM7 small interfering RNA (siRNA; 5'-UUCUCCGAACGUGUCACGUTT-3' , corresponding to coding region 1881-1901 of Rattus norvegicus TRPM7 mRNA (GeneID 679906) and a non-targeting siRNA control (5'-CCGGAGGUCAGGUCGAAAUTT- $3^{\prime}$ ) were synthesized by Genepharma (Shanghai, China). The AFs seeded in 12-well plates were transfected for $24 \mathrm{~h}$ with $50 \mathrm{~nm}$ of each siRNA using X-tremeGENE siRNA Transfection Reagent (Roche, Branchburg, NJ, USA) according to the manufacturer's instructions and were then stimulated with macrophage-conditioned medium for an additional $24 \mathrm{~h}$

\section{Western blotting}

AFs were harvested and protein was isolated using cell lysis buffer $(10 \mathrm{~mm}$ Tris-HCl, pH 8.0; $1 \mathrm{~mm}$ EDTA; 2\% SDS; $5 \mathrm{~mm}$ dithiothreitol; and $10 \mathrm{~mm}$ phenylmethanesulfonyl fluoride) containing freshly added protease inhibitor cocktail (Merck, Darmstadt, Germany). Protein concentrations were determined using the Bradford protein assay. The soluble lysates $(20 \mu \mathrm{g}$ per lane) were subjected to $8 \%$ SDS-PAGE for TRPM7, 12\% SDS-PAGE for SM22 -alpha and $10 \%$ SDS-PAGE for annexin- 1 and $\beta$-actin. Primary antibodies for TRPM7 (1:1000; Abcam), annexin-1 (1:1000; Santa Cruz Biotechnology) and SM-22- $\alpha$ (1:5000; Sigma-Aldrich, Shanghai, China) were incubated in 5\% nonfat milk for $1 \mathrm{~h}$ at room temperature. The levels of $\beta$-actin (1:6000; SigmaAldrich, St Louis, MO, USA) were analyzed to confirm equal loading. Relative protein levels were visualized using an enhanced chemiluminescence detection system (Pierce, Rockford, IL, USA).

\section{Statistical analyses}

Data are presented as the mean \pm s.d. Continuous variables were compared by either Student's $t$-test or an analysis of variance followed by the StudentNewman-Keuls post hoc test SPSS software, version 11.5 (SPSS Inc., Chicago, IL., USA). $P<0.05$ was considered statistically significant.
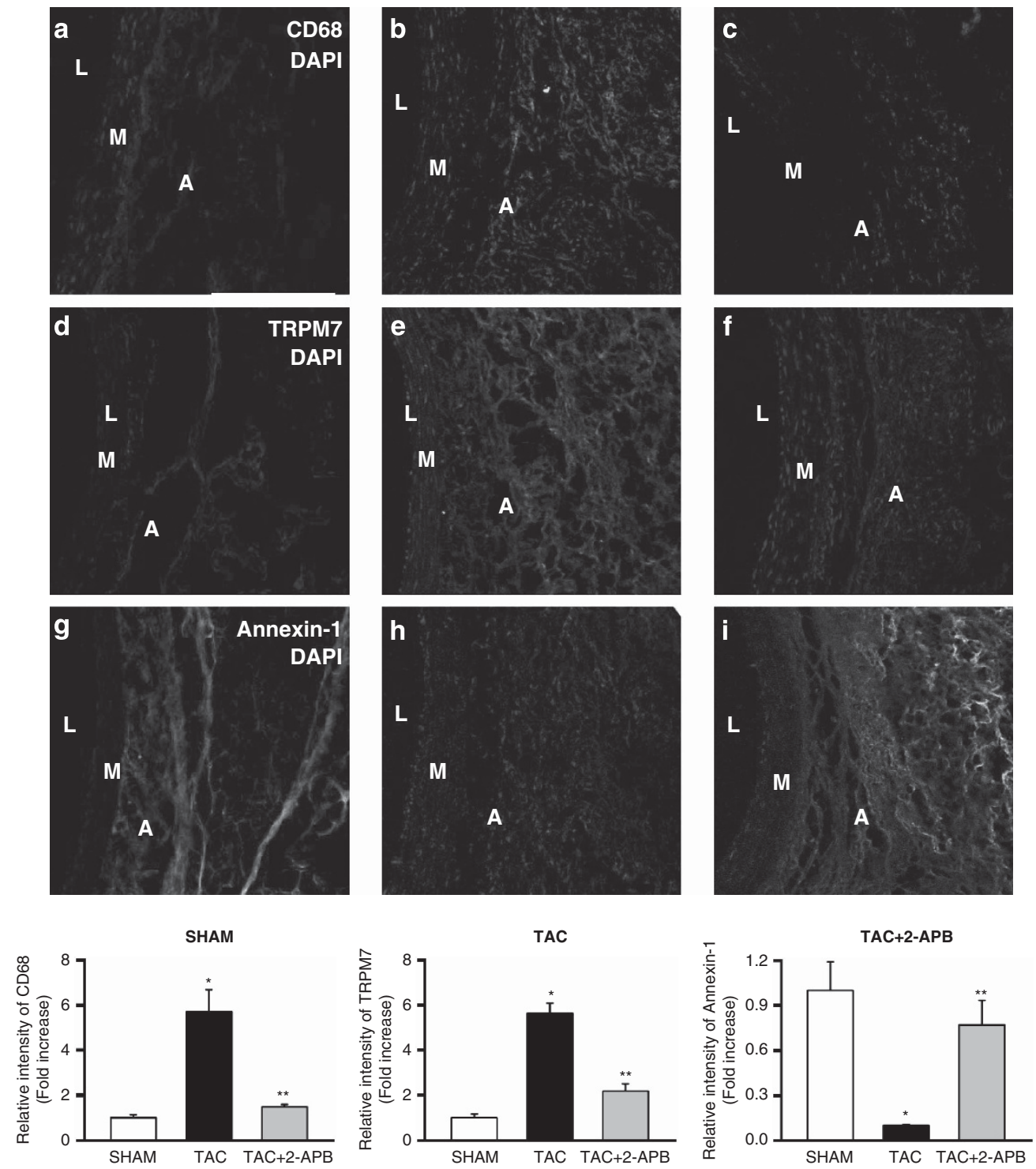

Figure 2 Immunofluorescence staining of CD68, transient receptor potential melastatin 7 (TRPM7) and annexin-1 in the SHAM (a,d,g), transverse aortic constriction (TAC; $\mathbf{b}, \mathbf{e}, \mathbf{h})$ and TAC treated with 2-aminoethoxydiphenyl borate (2-APB; $\mathbf{c}, \mathbf{f}, \mathbf{i})$ groups $(n=6)$. CD68 and TRPM7 were significantly increased in the adventitia of the right carotid arteries after TAC and were attenuated after treatment with 2-APB. Annexin-1 expression was markedly reduced and was subsequently rescued after 2-APB treatment. Nuclei were stained with 4',6-diamidino-2-phenylindole. a, adventitia; L, lumen; m, media. Bar, $100 \mu \mathrm{m}$. Results are expressed as the mean \pm s.d., ${ }^{*} P<0.05$ compared with the SHAM group. ${ }^{* *} P<0.05$ compared with the TAC group. A full color version of this figure is available at Hypertension Research online. 


\section{RESULTS}

Vascular remodeling in RCCA-B

Histomorphometric analysis revealed that pressure overload induced dramatic vessel thickening in RCCA-B 14 days after TAC compared with the sham-operated group (Figures 1a and b; Supplementary Table 1. The medial cross-section area was increased by $\sim 50 \%$ $(P<0.01$, Figure 1c). In addition, the adventitial cross-section area was increased by $\sim 125 \%(P<0.01$, Figure $1 \mathrm{~d})$. However, there was no significant difference in the left common carotid arteries compared with the sham group (data not shown).

TAC induced macrophage accumulation and TRPM7 upregulation in RCCA-B adventitia

An elevated expression of CD68, a specific phenotype marker for rat macrophages, ${ }^{27,28}$ was observed in the adventitia of RCCA-B (Figures $2 a$ and $b$ ), suggesting the accumulation of macrophages in TAC rats. In addition, TRPM7 was found to be upregulated in RCCA-B adventitia where macrophages accumulated in TAC rats (Figures $2 \mathrm{~d}$ and e). In this process, the expression of the TRPM7 downstream target, annexin-1, was diminished in RCCA-B (Figures $2 \mathrm{~g}$ and $\mathrm{h}$ ).

\section{Vascular inflammation and remodeling alterations by TRPM7 blocking}

To investigate the in vivo role of TRPM7 in vascular remodeling after TAC, the effects of 2-APB intraperitoneal injection on vascular adventitial inflammation in RCCA-B were examined. As shown in Figures $2 \mathrm{e}$ and $\mathrm{f}, 2$-APB treatment inhibited the upregulation of TRPM7 in TAC rats. Further, CD68, annexin-1 and MCP-1 levels were determined to test whether the blockade of TRPM7 regulated vascular adventitial inflammation in RCCA-B. Macrophage infiltration was significantly attenuated after 2-APB pretreatment compared with the TAC group (Figures $2 \mathrm{~b}$ and $\mathrm{c}$ ), and the downregulation of annexin- 1 by TAC was also rescued by 2 -APB (Figures $2 \mathrm{~h}$ and $\mathrm{i}$ ). The expression of another inflammatory factor, MCP-1, was induced by
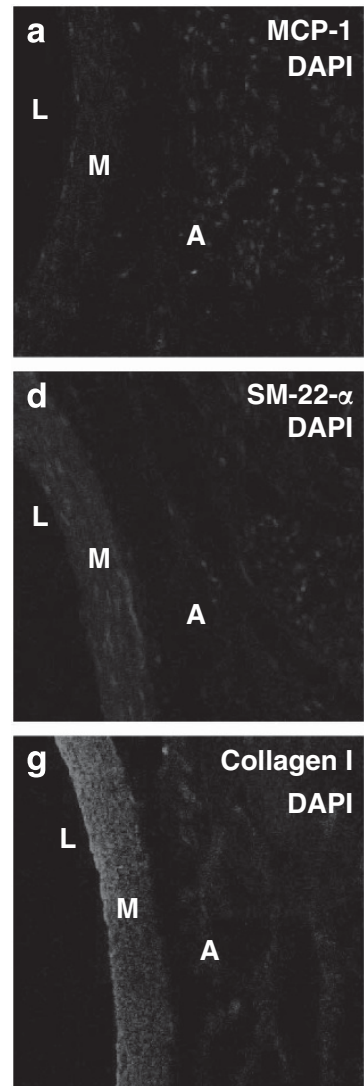

SHAM

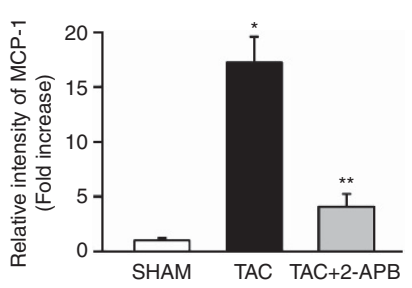

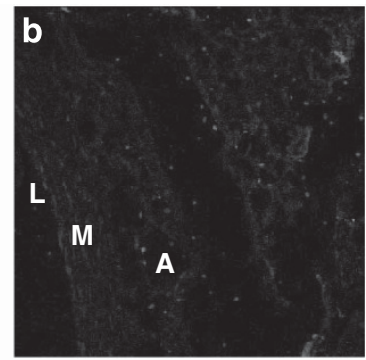
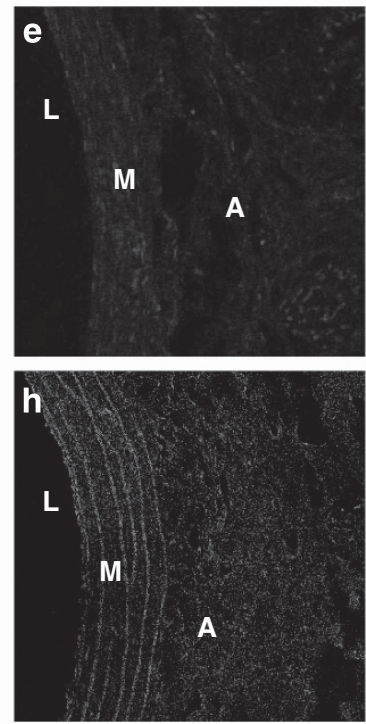

TAC

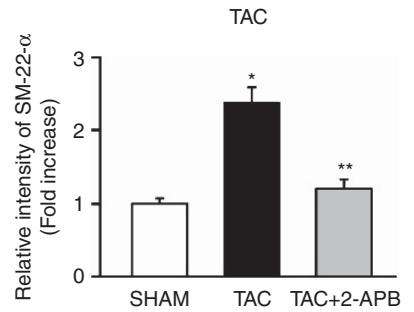

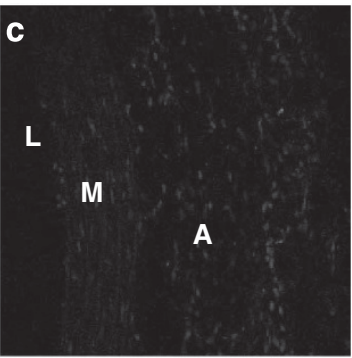
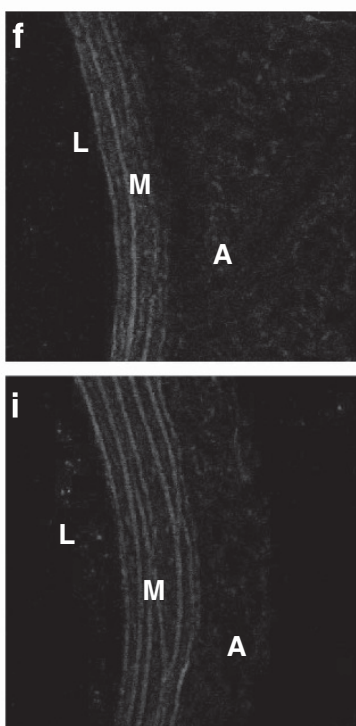

TAC+2-APB

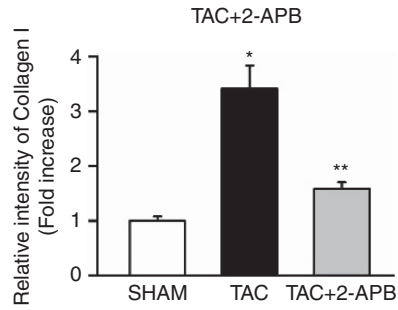

Figure 3 Immunoflurescence staining of monocyte chemotactic protein-1 (MCP-1), SM-22- $\alpha$ and collagen I in the SHAM (a,d,g), transverse aortic constriction (TAC; $\mathbf{b}, \mathbf{e}, \mathbf{h}$ ) and TAC treated with 2-aminoethoxydiphenyl borate (2-APB; $\mathbf{c}, \mathbf{f}, \mathbf{i})$ groups $(n=6)$. MCP-1, SM-22- $\alpha$ and collagen I expression were significantly increased in the adventitia of the right carotid arteries proximal to the band after TAC compared with the SHAM groups. 2-APB treatment inhibited MCP-1, SM-22- $\alpha$ and collagen I upregulation in TAC groups. Nuclei were stained with 4',6-diamidino-2-phenylindole. a, adventitia; L, lumen; m, media. Bar, $100 \mu \mathrm{m}$. Results are expressed as the mean \pm s.d., ${ }^{*} P<0.05$ compared with the SHAM group. ${ }^{*} P<0.05$ compared with the TAC group. A full color version of this figure is available at Hypertension Research online. 
pressure overload in RCCA-B (Figures $3 \mathrm{a}$ and $\mathrm{b}$ ) and reduced by 2 -APB treatment (Figure 3c). SM-22- $\alpha$ and collagen I levels were measured by immunostaining to further determine the effects of 2-APB on vascular remodeling process in RCCA-B. The blockade of TRPM7 attenuated the expression of MF phenotypic marker SM$22-\alpha$ (Shiz et al. ${ }^{29}$ ) under pressure overload in the adventitia of RCCA-B (Figures 3d-f), indicating that 2-APB treatment inhibited the transformation of AFs to MFs. In addition, abundant collagen I expression induced by TAC was significantly attenuated in the adventitia of RCCA-B after 2-APB treatment (Figures 3g-i).

Role of TRPM7 in the transformation from AFs to MFs

As mentioned above, TRPM7 was found to be related with the recruitment of macrophages after the TAC operation; however, the role of macrophages in vascular remodeling remains unclear. TRPM7 and macrophages mainly existed in the adventitia during remodeling, and thus the expression of SM-22- $\alpha$ was determined in primary rat vascular AFs pretreated with RAW264.7 macrophageconditioned medium by western blot analysis. The use of RAW264.7-conditioned medium increased the level of SM-22- $\alpha$ expression in a time- and dose-dependent manner (Figures 4 and 5). Interestingly, the expression of TRPM7 was increased, although the annexin-1 level was attenuated after pretreatment with macrophage-conditioned medium. TRPM7 blocker 2-APB was used in AFs to elucidate the role of TRPM7 in phenotypic modulation. It was observed that SM-22- $\alpha$ expression decreased after 2-APB pretreatment relative to incubation with RAW264.7-conditioned medium (Figure 4b). In addition to attenuating TRPM7, 2-APB lowered the expression of annexin-1 induced by macrophageconditioned medium (Figure 4b).

Further, cultured AFs were treated with specific TRPM7 siRNA or non-targeting siRNA control, and the expression of SM-22- $\alpha$ and annexin-1 was examined after RAW264.7-conditioned medium treatment for $24 \mathrm{~h}$. TRPM7 was shown to be required for the macrophage- a
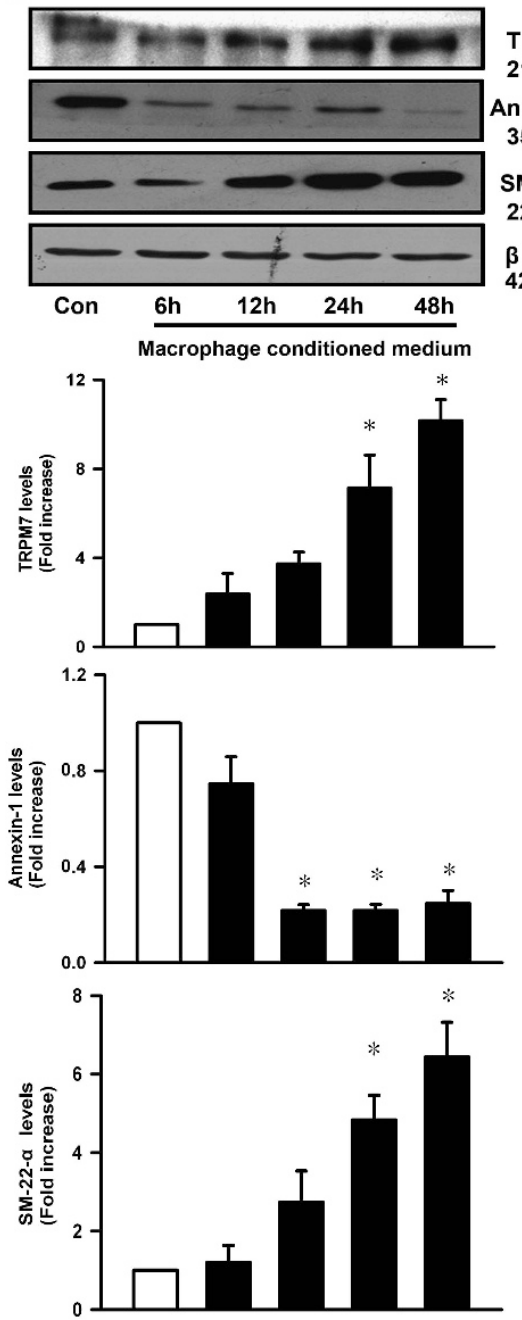

b
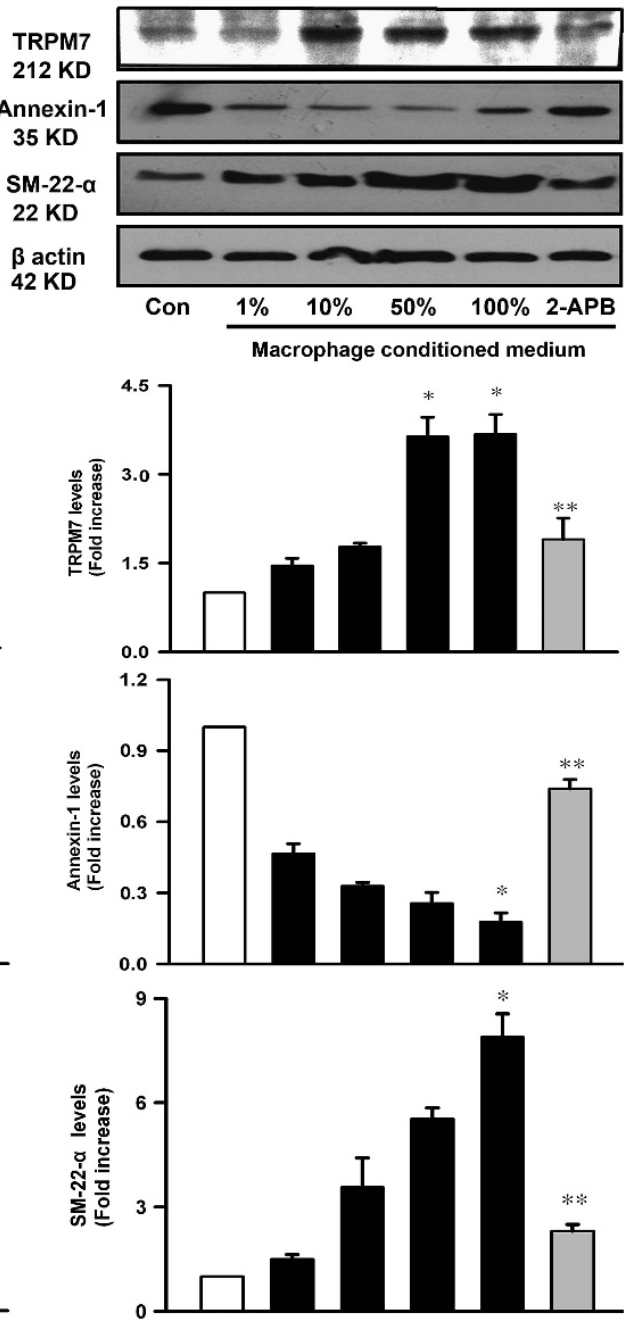

Figure 4 Western blot analysis of protein expression in adventitial fibroblasts. (a) Rat adventitial fibroblasts were treated with macrophage-conditioned medium for the indicated durations. (b) Adventitial fibroblasts were treated for $24 \mathrm{~h}$ with the indicated concentrations of macrophage-conditioned medium. To inhibit transient receptor potential melastatin 7 (TRPM7), adventitial fibroblasts were pretreated with $100 \mu \mathrm{M}$ 2-aminoethoxydiphenyl borate (2-APB) for $1 \mathrm{~h}$ and then stimulated with $100 \%$ macrophage-conditioned medium for $24 \mathrm{~h}$. The upper panel shows representative immunoblots of TRPM7, annexin- 1 and SM-22- $\alpha$ expression in adventitial fibroblasts. $\beta$-actin served as the internal control. The lower panels show the quantification of bands by densitometry. Results are shown as fold increases relative to the control for three independent experiments. Data are the mean \pm s.d. ${ }^{*} P<0.05$ compared with the control. ${ }^{* *} P<0.05$ compared with $100 \%$ macrophage-conditioned medium. 
conditioned medium-triggered phenotypic modulation of AFs and downregulation of annexin-1 (Figure 5).

\section{DISCUSSION}

Our findings demonstrated that in vivo macrophages accumulated in the remodeled adventitia of RCCA-B under pressure overload, and in vitro they precipitated the transformation of AFs to MFs. Strikingly, a novel molecule related to pressure overload was discovered in our study; TRPM7 can mediate the pressure overload-induced macrophage accumulation and affect the expression of MCP-1 and annexin-1 in RCCA-B. Further, TRPM7 participated in vascular AFs transformation and increased collagen I formation in vivo. Macrophages further led to AFs transformation, elevated TRPM7 and reduced annexin-1, all of which were normalized by the addition of 2-APB and anti-TRPM7 siRNA.

It was once believed that adventitia is a contributor to vascular function as a support tissue; however, there is increasing evidence that adventitia has a crucial role in several vascular diseases, such as hypertension, atherosclerosis and restenosis. ${ }^{3}$ The phenotypic transformation of AFs to MFs contributes to neointimal formation in injured porcine coronary arteries ${ }^{30}$ and adventitial remodeling in thickened aorta and the right carotid in the TAC model. ${ }^{8,9}$ In view of the similarity between arteries proximal to the band in the TAC model and arteries under pressure overload in human hypertension, as well as the widespread application of the TAC model in left ventricular hypertrophy and heart failure, ${ }^{23,31}$ we suppose that the model presented here is appropriate. As expected, greater than onefold thickening of adventitia was detected at 14 days post TAC operation. Moreover, in agreement with previous reports, ${ }^{8,9}$ we observed increases in macrophage infiltration as well as SM-22- $\alpha$, MCP-1 and collagen I levels in the TAC model, indicating the reliability of our experiments.

TRPM7 has been implicated in the dysfunction of endothelial cells and vascular smooth muscle cells, ${ }^{12,22,32-34}$ although its expression and function remain poorly understood in vascular adventitia. It is noteworthy that reduction of annexin-1, a substrate of TRPM7, was observed in pressure overload-induced adventitial remodeling as well as in MFs transformation caused by macrophage-conditioned medium. We also observed this reduction in AFs and the aorta of spontaneously hypertensive rats in a previous study. ${ }^{19}$ Thus, we further established the pathophysiological significance of TRPM7 in TAC rats by demonstrating an increased expression of TRPM7 in vascular adventitia, where inflammation and remodeling occurred. Further treatment with TRPM7 inhibitor 2-APB ${ }^{35,36}$ was observed to reverse the changes in inflammation factors and molecules relevant to vascular remodeling under pressure overload. Taken together, these findings suggest that TRPM7 could mediate pathological alteration in the vascular adventitia. It has been reported that the TRPM7 current can be activated by stretching or shear stress in vascular smooth muscle cells and other cells, supporting its role in response to vessel injury. ${ }^{37-39}$ Therefore, we present the hypothesis that the activation of TRPM7 by pressure overload-induced stretching can recruit macrophages into thickened adventitia and increase remodeling in a mechanosensitive manner.

To determine whether inflammation and remodeling in vascular adventitial are correlated, we explored the role of macrophageconditioned medium in the phenotypic modulation of AFs. We found that macrophages were involved in vascular remodeling via the triggering of MF differentiation. Interestingly, exposure to macrophage-conditioned medium led to an upregulation of the expression of TRPM7, whereas this process companied with MF differentiation
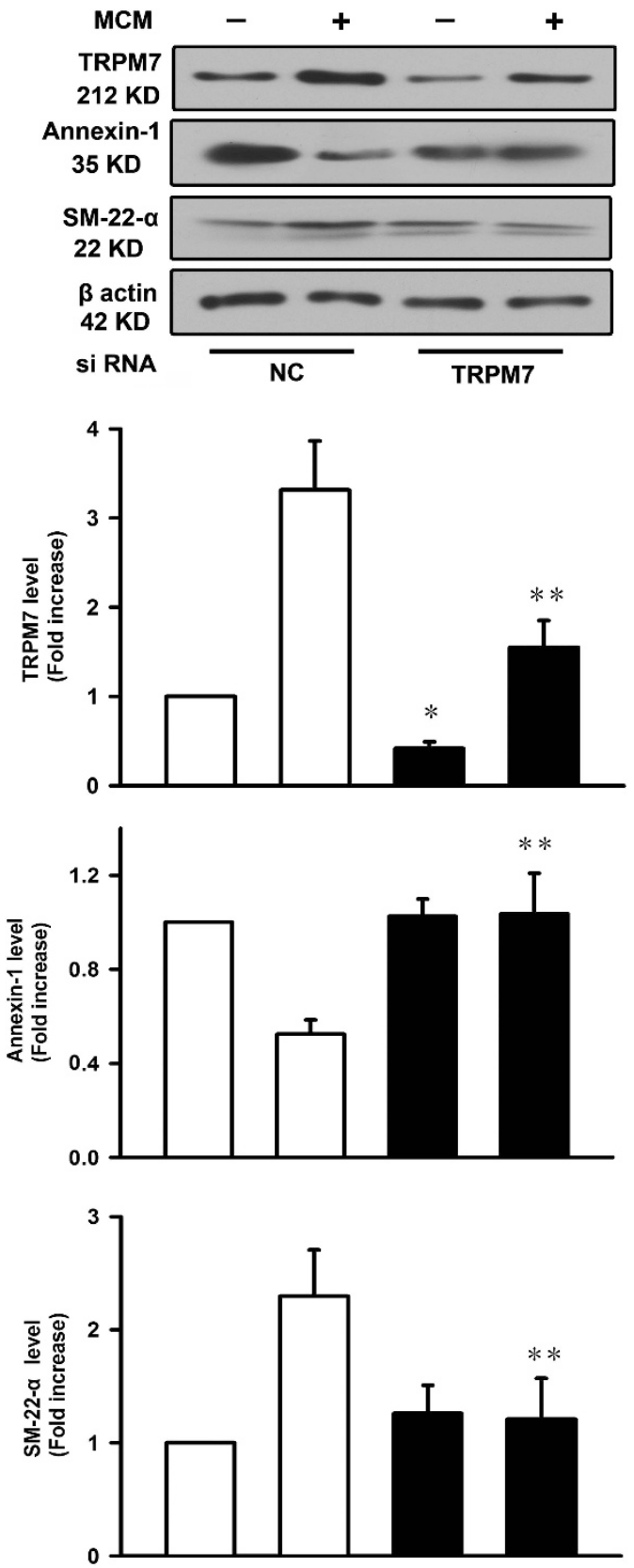

Figure 5 Small interfering RNA (siRNA)-mediated repression of transient receptor potential melastatin 7 (TRPM7) induced adventitial fibroblasts (AFs) transformation and increased expression of annexin-1. The adventitial fibroblasts were transfected with siRNA for $24 \mathrm{~h}$ and then stimulated with macrophage-conditioned medium for an additional $24 \mathrm{~h}$. The upper panel shows representative immunoblots of TRPM7, annexin-1 and SM-22- $\alpha$ expression in adventitial fibroblasts. $\beta$-actin served as an internal control. The lower panels show the quantification of bands by densitometry. The results are shown as fold increases relative to the control for three independent experiments. Data are the mean \pm s.d. ${ }^{*} P<0.05$ compared with the non-targeting siRNA control (NC). ${ }^{* *} P<0.05$ compared with NC treated with macrophage-conditioned medium.

was inhibited by the addition of 2-APB. 2-APB is typically reported to inhibit the TRPM7 channel activity. In this present study, we found that 2-APB suppressed the expression of TRPM7 triggered by pressure overload and exposure to the macrophage-conditioned medium, 
which is consistent with the observations of Liu $\mathrm{H}$ et al. ${ }^{35}$ As a blocker of store-operated Ca entry, ${ }^{40}$ 2-APB may regulate TRPM7 expression by altering $\mathrm{Ca}$ storage and/or release, which are involved in processes related to the regulation of gene expression. Otherwise, to examine a more specific effect of TRPM7 on AFs phenotype switching in vitro, we pretreated the cultured AFs with anti-TRPM7 siRNA before being stimulated with RAW264.7-conditioned medium. We found that the suppression of TRPM7 partly normalized the changes in AF gene expression (SM-22- $\alpha$ and annexin-1) initiated by the macrophageconditioned medium, further supporting the hypothesis that TRPM7 is required for cell phenotype switching in AFs exposing to the macrophage-conditioned medium. On the basis of this line of evidence, it is reasonable to speculate that by recruiting inflammation cells, TRPM7 might interact with macrophages to participate in vascular remodeling.

TRPM7 is vital for embryonic development, and its global disruption in prenatal mice is lethal. ${ }^{41}$ The siRNA of TRPM7 can inhibit its activities in vitro but not in vivo (data not shown). Considering the similar effects between 2-APB and the siRNA of TRPM7 on in vitro phenotypic modulation of AFs and as suggested by other in vivo studies, ${ }^{20,42,43}$ we employed 2-APB to block the effect of TRPM7 in vivo in this study. However, on a micromolar scale, 2-APB can increase TRPM6 to a maximal extent but significantly inhibit TRPM7 channel activities, whereas on a millimolar scale, it can potentiate TRPM6/7 and TRPM7 channel activities. ${ }^{44}$ Here, we acknowledge that 2-APB is a nonspecific inhibitor of TRPM7, which may present a limitation of this study in vivo.

In summary, our findings demonstrate that TRPM7 modulates the infiltration of macrophages and acts synergistically to promote collagen accumulation and vascular adventitial MF formation during pressure overload in TAC rats. To the best of our knowledge, this is the first description of the potential regulatory roles of TRPM7 in vascular inflammation and pressure overload-mediated vascular adventitial collagen accumulation and cell phenotypic transformation. These data suggest that the targeting of TRPM7 has potential therapeutic importance for vascular diseases.

\section{ACKNOWLEDGEMENTS}

This work was supported by the National Natural Science Foundation of China (81370431, 81230071 and 81170246), the Science Fund of Shanghai Jiao Tong University School of Medicine (11XJ21034) and the Shanghai Pujiang Talents Program of Shanghai Science and Technology Committee (11PJ1408300).

1 Bots ML, Dijk JM, Oren A, Grobbee DE. Carotid intima-media thickness, arterial stiffness and risk of cardiovascular disease: current evidence. J Hypertens 2002; 20: 2317-2325.

2 Laurent S. Arterial wall hypertrophy and stiffness in essential hypertensive patients. Hypertension 1995; 26: 355-362.

3 Sartore S, Chiavegato A, Faggin E, Franch R, Puato M, Ausoni S, Pauletto P. Contribution of adventitial fibroblasts to neointima formation and vascular remodeling: from innocent bystander to active participant. Circ Res 2001; 89: 1111-1121.

4 Shi Y, Pieniek M, Fard A, O'Brien J, Mannion JD, Zalewski A. Adventitial remodeling after coronary arterial injury. Circulation 1996; 93: 340-348.

5 Li G, Chen SJ, Oparil S, Chen YF, Thompson JA. Direct in vivo evidence demonstrating neointimal migration of adventitial fibroblasts after balloon injury of rat carotid arteries. Circulation 2000; 101: 1362-1365.

6 Siow RC, Mallawaarachchi CM, Weissberg PL. Migration of adventitial myofibroblasts following vascular balloon injury: insights from in vivo gene transfer to rat carotid arteries. Cardiovasc Res 2003; 59: 212-221.

7 Li YH, Hsieh CY, Wang DL, Chung HC, Liu SL, Chao TH, Shi GY, Wu HL. Remodeling of carotid arteries is associated with increased expression of thrombomodulin in a mouse transverse aortic constriction model. Thromb Haemost 2007; 97: 658-664.

8 Eberth JF, Gresham VC, Reddy AK, Popovic N, Wilson E, Humphrey JD. Importance of pulsatility in hypertensive carotid artery growth and remodeling. J Hypertens 2009; 27: 2010-2021.
9 Chen J, Wu J, Li L, Zou YZ, Zhu DL, Gao PJ. Effect of an acute mechanical stimulus on aortic structure in the transverse aortic constriction mouse model. Clin Exp Pharmacol Physiol 2011; 38: 570-576.

10 Nadler MJ, Hermosura MC, Inabe K, Perraud AL, Zhu Q, Stokes AJ, Kurosaki T, Kinet JP, Penner R, Scharenberg AM, Fleig A. LTRPC7 is a Mg.ATP-regulated divalent cation channel required for cell viability. Nature 2001; 411: 590-595.

11 Runnels LW, Yue L, Clapham DE. TRP-PLIK, a bifunctional protein with kinase and ion channel activities. Science 2001; 291: 1043-1047.

12 Touyz RM. Transient receptor potential melastatin 6 and 7 channels, magnesium transport, and vascular biology: implications in hypertension. Am J Physiol Heart Circ Physiol 2008; 294: H1103-H1118.

13 Yogi A, Callera GE, Antunes TT, Tostes RC, Touyz RM. Transient receptor potential melastatin 7 (TRPM7) cation channels, magnesium and the vascular system in hypertension. Circ J 2011; 75: 237-245.

14 Yogi A, Callera GE, Tostes R, Touyz RM. Bradykinin regulates calpain and proinflammatory signaling through TRPM7-sensitive pathways in vascular smooth muscle cells. Am J Physiol Regul Integr Comp Physiol 2009; 296: R201-R207.

15 Callera GE, He Y, Yogi A, Montezano AC, Paravicini T, Yao G, Touyz RM. Regulation of the novel Mg2 + transporter transient receptor potential melastatin 7 (TRPM7) cation channel by bradykinin in vascular smooth muscle cells. J Hypertens 2009; 27: $155-166$.

16 Clark K, Langeslag M, van Leeuwen B, Ran L, Ryazanov AG, Figdor CG, Moolenaar WH, Jalink K, van Leeuwen FN. TRPM7, a novel regulator of actomyosin contractility and cell adhesion. EMBO J 2006; 25: 290-301.

17 Dorovkov MV, Ryazanov AG. Phosphorylation of annexin I by TRPM7 channel-kinase. J Biol Chem 2004; 279: 50643-50646.

18 La M, Tailor A, D'Amico M, Flower RJ, Perretti M. Analysis of the protection afforded by annexin 1 in ischaemia-reperfusion injury: focus on neutrophil recruitment. Eur $J$ Pharmacol 2001; 429: 263-278.

19 Guo SJ, Wang TR, Chen J, Wu LY, Gao PJ, Zhu DL. Dynamic expression of proteins associated with adventitial remodeling in adventitial fibroblasts from spontaneously hypertensive rats. Acta Pharmacol Sin 2010; 31: 1312-1318.

20 Inoue K, Xiong ZG. Silencing TRPM7 promotes growth/proliferation and nitric oxide production of vascular endothelial cells via the ERK pathway. Cardiovasc Res 2009; 83: 547-557.

21 Montezano AC, Zimmerman D, Yusuf H, Burger D, Chignalia AZ, Wadhera V, van Leeuwen FN, Touyz RM. Vascular smooth muscle cell differentiation to an osteogenic phenotype involves TRPM7 modulation by magnesium. Hypertension 2010; 56: 453-462.

22 Paravicini TM, Yogi A, Mazur A, Touyz RM. Dysregulation of vascular TRPM7 and annexin-1 is associated with endothelial dysfunction in inherited hypomagnesemia. Hypertension 2009; 53: 423-429.

23 Rockman HA, Ross RS, Harris AN, Knowlton KU, Steinhelper ME, Field LJ, Ross J Jr, Chien KR. Segregation of atrial-specific and inducible expression of an atrial natriuretic factor transgene in an in vivo murine model of cardiac hypertrophy. Proc Natl Acad Sci USA 1991; 88: 8277-8281.

24 Faber JE, Yang N, Xin X. Expression of alpha-adrenoceptor subtypes by smooth muscle cells and adventitial fibroblasts in rat aorta and in cell culture. J Pharmacol Exp Ther 2001; 298: 441-452.

25 Shen WL, Gao PJ, Che ZQ, Ji KD, Yin M, Yan C, Berk BC, Zhu DL. NAD(P)H oxidasederived reactive oxygen species regulate angiotensin-II induced adventitial fibroblast phenotypic differentiation. Biochem Biophys Res Commun 2006; 339: 337-343.

26 Macianskiene R, Martisiene I, Zablockaite D, Gendviliene V. Characterization of Mg2 +-regulated TRPM7-like current in human atrialmyocytes. J Biomed Sci 2012; 19: 75.

27 Chiavegato A, Bollini S, Pozzobon M, Callegari A, Gasparotto L, Taiani J, Piccoli M, Lenzini E, Gerosa G, Vendramin I, Cozzi E, Angelini A, Iop L, Zanon GF, Atala A, De Coppi $P$, Sartore S. Human amniotic fluid-derived stem cells are rejected after transplantation in the myocardium of normal, ischemic, immuno-suppressed or immuno-deficient rat. J Mol Cell Cardiol 2007; 42: 746-759.

28 Badylak SF, Valentin JE, Ravindra AK, McCabe GP, Stewart-Akers AM. Macrophage phenotype as a determinant of biologic scaffold remodeling. Tissue Eng Part A 2008; 14: 1835-1842.

29 Shi ZD, Abraham G, Tarbell JM. Shear stress modulation of smooth muscle cell marker genes in 2-D and 3-D depends on mechanotransduction by heparan sulfate proteoglycans and ERK1/2. PLoS One 2010; 5: e12196.

30 Shi Y, O'Brien JE, Fard A, Mannion JD, Wang D, Zalewski A. Adventitial myofibroblasts contribute to neointimal formation in injured porcine coronary arteries. Circulation 1996; 94: 1655-1664.

31 Beckles DL, Mascareno E, Siddiqui MA. Inhibition of Jak2 phosphorylation attenuates pressure overload cardiac hypertrophy. Vascul Pharmacol 2006; 45: 350-357.

32 Gurney AM. Going with the flow: smooth muscle TRPM7 channels and the vascular response to blood flow. Circ Res 2006; 98: 163-164.

33 Touyz RM, He Y, Montezano AC, Yao G, Chubanov V, Gudermann T, Callera GE. Differential regulation of transient receptor potential melastatin 6 and 7 cation channels by ANG II in vascular smooth muscle cells from spontaneously hypertensive rats. Am J Physiol Regul Integr Comp Physiol 2006; 290: R73-R78.

34 Zhang Z, Wang M, Fan XH, Chen JH, Guan YY, Tang YB. Upregulation of TRPM7 channels by angiotensin II triggers phenotypicswitching of vascular smooth muscle cells of ascending aorta. Circ Res 2012; 111: 1137-1146. 
35 Liu H, Li J, Huang Y, Huang C. Inhibition of transient receptor potentia melastain 7 channel increases HSCs apoptosis induced by TRAIL. Life Sci 2012 90: 612-618.

36 Zhang YH, Sun HY, Chen KH, Du XL, Liu B, Cheng LC, Li X, Jin MW, Li GR. Evidence for functional expression of TRPM7 channels in human atrial myocytes. Basic Res Cardiol 2012; 107: 282

37 Inoue R, Jian Z, Kawarabayashi Y, Mechanosensitive TRP. channels in cardiovascular pathophysiology. Pharmacol Ther 2009; 123: 371-385.

38 Numata T, Shimizu T, Okada Y. TRPM7 is a stretch- and swelling-activated cation channel involved in volume regulation in human epithelial cells. Am J Physiol Cell Physiol 2007; 292: C460-C467.

39 Oancea E, Wolfe JT, Clapham DE. Functional TRPM7 channels accumulate at the plasma membrane in response to fluid flow. Circ Res 2006; 98: 245-253.
40 Prakriya M, Lewis RS. Potentiation and inhibition of $\mathrm{Ca}(2+)$ release-activated $\mathrm{Ca}(2+)$ channels by 2-aminoethyldiphenyl borate (2-APB) occurs independently of IP(3) receptors. J Physiol 2001; 536: 3-19.

41 Jin J, Desai BN, Navarro B, Donovan A, Andrews NC, Clapham DE. Deletion of Trpm7 disrupts embryonic development and thymopoiesis without altering Mg2 + homeostasis. Science 2008; 322: 756-760.

42 Lu Y, Wu X, Dong Y, Xu Z, Zhang Y, Xie Z. Anesthetic sevoflurane causes neurotoxicity differently in neonatal naive and Alzheimer disease transgenic mice. Anesthesiology 2010; 112: 1404-1416.

43 Nicoud IB, Knox CD, Jones CM, Anderson CD, Pierce JM, Belous AE, Earl TM, Chari RS 2-APB protects against liver ischemia-reperfusion injury by reducing cellular and mitochondrial calcium uptake. Am J Physiol Gastrointest Liver Physiol 2007; 293: G623-G630.

$44 \mathrm{Li} \mathrm{M}$, Jiang J, Yue L. Functional characterization of homo- and heteromeric channel kinases TRPM6 and TRPM7. J Gen Physiol 2006; 127: 525-537.

Supplementary Information accompanies the paper on Hypertension Research website (http://www.nature.com/hr) 